\title{
Survey on the Readiness for Hospital Discharge and Its Influencing Factors among Patients with Cardiac Valve Replacement
}

\author{
Limin Liang1, Youdi Cai2 ${ }^{*}$, Jiaojiao Gu², Huan $\mathrm{Li}^{2}$, Shiju Huang², Hengying Fang \\ ${ }^{1}$ Department of Cardiothoracic Surgery, The Third Affiliated Hospital of Sun Yat-sen University, Guangzhou, China \\ ${ }^{2}$ Department of Surgery, The Third Affiliated Hospital of Sun Yat-sen University, Guangzhou, China \\ ${ }^{3}$ Department of Nursing, The Third Affiliated Hospital of Sun Yat-sen University, Guangzhou, China \\ Email: ${ }^{\star} 417192990 @ q q . c o m$
}

How to cite this paper: Liang, L.M., Cai, Y.D., Gu, J.J., Li, H., Huang, S.J. and Fang, H.Y. (2021) Survey on the Readiness for Hospital Discharge and Its Influencing Factors among Patients with Cardiac Valve Replacement. Open Journal of Nursing, 11, 302-314.

https://doi.org/10.4236/ojn.2021.115027

Received: March 12, 2021

Accepted: May 15, 2021

Published: May 18, 2021

Copyright $\odot 2021$ by author(s) and Scientific Research Publishing Inc. This work is licensed under the Creative Commons Attribution International License (CC BY 4.0).

http://creativecommons.org/licenses/by/4.0/

\begin{abstract}
Background: There are about 200,000 patients in China who need to undergo valve replacement surgery each year. With the promotion and application of the Fast-Track Surgery concept and the increase in hospital bed turnover rate, the average hospital stay of patients undergoing CVR surgery has been significantly shortened, and thus the patients are still in the recovery stage rather than fully recovered when they are discharged from the hospital. Good preparation for discharge can improve patients' post-discharge self-care ability, reduce the risk of re-admission, and save medical resources. Objective: To describe the status of readiness for hospital discharge among postoperative patients that have undergone CVR, and to explore its influencing factors. Methods: The general information questionnaire, the Readiness for Hospital Discharge Scale and the Quality of discharge Teaching Scale were utilized to investigate 130 post-CVR patients admitted to the Cardiothoracic Surgery Department of a tertiary A-Class hospital in Guangzhou from July 2019 to October 2020. Results: The Readiness for Hospital Discharge Scale was at a moderate level with a total score of $163.88 \pm 39.082$, while the Quality of Discharge Teaching Scale was also at a moderate level with a total score of 194.09 \pm 40.643. Multiple linear regression analysis revealed that the quality of discharge guidance, gender, and occupation were the influencing factors of CVR patient's readiness for hospital discharge. These three variables jointly explained $45.8 \%$ of the total variation. Conclusion: The level of CVR patients' readiness for hospital discharge is at a moderate level, and the quality of discharge guidance positively affects patients' readiness for discharge. Therefore, in clinical work, attention should be paid to patient discharge guidance. Personalized health education should be implemented to improve the quality of patient guidance.
\end{abstract}




\section{Keywords}

Cardiac Valve Replacement, Patient Discharge, Readiness for Hospital

Discharge, Influencing Factors Analysis

\section{Introduction}

Cardiac Valve Replacement (CVR) has become the most effective treatment for acquired or congenital heart valve diseases [1]. Every year, more than 450,000 patients worldwide undergo valve replacement surgery [2]. The development of heart valve surgery in China has a history of more than 50 years, and about 200,000 patients need to undergo valve replacement surgery every year [3]. With the promotion and application of the Fast-Track Surgery concept and the increase in hospital bed turnover rate, the average hospital stay of patients undergoing CVR surgery has been significantly shortened, and thus the patients are still in the recovery stage rather than fully recovered when they are discharged from the hospital [4] [5]. Under this healthcare situation, how to not only ensure the safety of patients when discharged from the hospital but also ensure that patients and their families have the ability to undertake follow-up care after discharge has become a key issue. Discharge readiness refers to medical staff's comprehensive assessment of the patient's ability to further recover after leaving the medical institution, taking the patient's physical, psychological and social health status all into consideration. It indicates the patient's perception of whether he or she is ready for hospital discharge, and it is an indicator suggesting that the patient has an adequate level of recovery to be discharged safely (although not fully recovered but has been effectively recovering) [6] [7]. This study intends to explore the general situation of patients' discharge readiness after CVR, and discuss its related factors, aiming to improve patients' discharge readiness, ensure patients' safe discharge, improve patients' post-discharge self-care ability, reduce the risk of re-admission, and save healthcare resources.

\section{Subjects and Methods}

\subsection{Subjects}

Convenience sampling was adopted to select patients who had performed valve replacement in a tertiary hospital in Guangzhou from July 2019 to October 2020. Inclusion criteria: 1) implemented valve replacement surgery; 2) discharged patients who have reached the discharge standard after hospitalization; 3) understand the purpose, procedures and content of the study, voluntarily participate in the trial and sign the informed consent; 4) proficient in Mandarin or Cantonese, able to communicate effectively with the researchers; 5) age $\geq 18$ years old. Exclusion criteria: 1) patients with dysgnosia or mental abnormality; 2) those who are too weak to complete the questionnaire survey; 3) those who are unconscious, refuse to cooperate, or express unclearly; 4) those who cannot com- 
municate effectively with the researchers owing to literacy level, hearing or mentality problems, etc. According to the principles of scale design and psychometrics, one item in the scale corresponds to the size of $5-10$ samples. Therefore, the sample size should be at least $\mathrm{N} \times 5$, where $\mathrm{N}$ refers to the number of item entries; meanwhile, considering that unreturned and invalid questionnaires could take up about $10 \%-20 \%$, the total sample size should be at least ( $\mathrm{N}$ $\times 5) \times 110 \%$ cases. In this study, the preset number of item entries was 23 , so 130 samples were required.

\subsection{Research Tools}

\subsubsection{General Information Questionnaire}

The questionnaire was designed and completed by the members of the research team through literature review and group discussion. It was divided into two parts: the first part was demographic sociology information, including gender, age, marital status, literacy level, working status, habitation manner, family monthly income and medical expenses payment, etc.; the second part was disease/treatment-related information, including hospitalization days, disease onset situation and the number of hospitalizations, changes after returning home due to the disease, the number of complication types and the number of drugs taken, etc.

\subsubsection{Readiness for Hospital Discharge Scale (RHDS)}

The scale was developed by Weiss et al. in the United States in 2006 [8], including two versions, namely the patient-perceived discharge readiness scale (PT-RHDS) and nurse-perceived discharge readiness scale (NT-RHDS), evaluating the patient's readiness for discharge from the perspective of the patient and the nurse, respectively. Among them, the patient-perceived discharge readiness scale is the most widely used one. After confirmatory factor analysis, control group comparison and predictive validity test, it was confirmed that the scale structure, which included 4 dimensions and 21 items, presented reasonable validity; the content consistency test results showed that the Cronbach's $\alpha$ coefficient of the entire scale was 0.90 . The scale has been widely used internationally to assess the readiness for discharge of patients with different diseases. The Cronbach's $\alpha$ coefficient of the entire scale ranges from 0.84 to 0.92 in different populations, showing good reliability. This study used the Chinese version of RHDS [9] which was translated and revised by Zhao Huiling and others from West China Hospital of Sichuan University. The Chinese version of the scale contains 23 items, consisting of 4 dimensions: one's self-condition, disease knowledge, coping ability after discharge, and available social support. The scale is a self-rating summation level scale, and the score of each item ranges from 0 to 10 points. The research subjects choose the score that suits them according to the description under the score. The scale's total score equals to the sum of scores in the 4 dimensions; the higher the score, the better the patient's readiness for discharge. The total Cronbach's $\alpha$ coefficient of the scale is 0.929 , and the Cronbach's $\alpha$ 
coefficient of each dimension is $0.824-0.901$.

\subsubsection{Quality of Discharge Teaching Scale (QDTS)}

The original scale was developed by Weiss et al. Wang Binghua et al. [10] obtained the translation permission and use authorization from the original scale author in 2015 and translated it into Chinese. The Chinese version of the QDTS scale includes 3 dimensions: required content (6 items), received content (6 items), as well as instructional skills and effects ( 12 items). The items are based on a 0 - 10 scale scoring method, assigned from "not at all/never" to "very much/always". The 12 items in the first two dimensions form a paired 6 groups of items. By comparing the content scores received and required by the patient, the researchers can understand whether the given discharge guidance content meets the needs of the patient. The entire scale measures the treatment of discharge guidance by calculating the total scores of the two dimensions of "received content" and "instructional skills and effects". The higher the total score, the better the quality of discharge guidance. The scale has good reliability and validity. The total Cronbach's $\alpha$ coefficient is 0.924 , and the Cronbach's $\alpha$ coefficient of each dimension is $0.882-0.935$.

\section{Method}

\subsection{Pre-Survey}

In this study, 17 patients were selected for pre-survey tests. The investigator first distributed the questionnaire, and then asked and recorded their views on the questionnaire after the patients had completed the questionnaire. The research group adjusted the questionnaire after discussing the results of the preliminary tests.

\subsection{Data Collection Methods}

1) Obtain the consent and support of the relevant departments' directors; 2) Explain the purpose and significance of the research to the respondents before issuing the questionnaire, following the principle of voluntariness and recording the questionnaire anonymously; 3) Strictly follow the sample inclusion and exclusion criteria, and conduct the questionnaire survey on the enrolled subject on the day when he or she was discharged from the hospital; 4) Use a fixed questionnaire with unified instruction and filling methods, and explain to the subjects how to fill in the questionnaire; 5) Let the surveyed subjects complete the questionnaire as required; 6) For patients who were illiterate or had limitations in activities, the investigator read each item for the patients and fill out the questionnaire based on the patient's answers; 7) After the patient filled out the questionnaire, carefully check the questionnaire, and complete the answers at the scene if there were any vacancies or omissions; 8) Evaluate the returned questionnaires and remove some invalid questionnaires. In this study, a total of 141 questionnaires were distributed and 130 valid questionnaires were returned. The effective response rate was $92.2 \%$. 


\subsection{Statistical Methods}

The data was entered into Excel to establish a database, and SPSS 19.0 software was used for statistical analysis. The general data of patients were described by frequency, percentage, mean and standard deviation. The sample size of this study was large, which could be regarded as approximately normally distributed data; the dimensions of the scale and the score of the total scale were described by the mean \pm standard deviation $(\bar{x} \pm s)$. In univariate analysis, two independent sample t-tests were used for binary categorical variables, single-factor analysis of variance was used for multi-categorical variables that meet the homogeneity of variance, while nonparametric test (Kruskal-Wallis $\mathrm{H}$ test) was used for the contrary cases. Pearson correlation analysis was performed on the discharge readiness and the quality of discharge guidance, and multiple linear regression was used for multivariate analysis.

\section{Results}

\subsection{Current Status of Discharge Readiness and Discharge Guidance Quality of Patients after Cardiac Valve Surgery}

This study investigated 130 post-CVR patients. Since the number of items in each dimension of the scale was different, and the range of scores was inconsistent, the dimension scores were standardized: standardized score $=$ dimension total score/number of items. According to the statistics of item entry 1, 119 patients (91.54\%) thought they were ready to be discharged from the hospital, while the other 11 patients $(8.46 \%)$ thought they were not ready. The total score of patients' discharge readiness was $163.88 \pm 39.082$ points, which was at a medium level, and the total score of discharge guidance quality was $194.09 \pm 40.643$ points, which was also at a medium level. The total scores of patients' post-CVR discharge readiness, the scores of various dimensions and the status of discharge guidance quality scores are shown in Table 1 and Table 2.

\subsection{Comparison of Discharge Readiness Scores of Patients after Cardiac Valve Surgery with Different Demographic Characteristics}

There were statistical differences in the scores of patients with different occupations in hospital discharge readiness, among which the unemployed had the highest score. The distance between home and the hospital was also one of the factors that affected the readiness for discharge. Patients within $10 \mathrm{~km}$ from the home had a higher level of readiness for discharge, as shown in Table 3.

\subsection{Correlation Analysis of Discharge Readiness and Discharge Guidance Quality of Patients after Cardiac Valve Surgery}

There was a positive correlation between the patient's discharge readiness score and the quality of discharge quality guidance (required content, received content, instructional skills and effects), as shown in Table 4. 
Table 1. Discharge readiness scores of patients after cardiac valve surgery.

\begin{tabular}{ccccc}
\hline Dimension & No. of Entries & Full Score & Actual Score & Standardized Score \\
\hline Self-condition & 7 & 70 & $51.58 \pm 11.261$ & $7.38 \pm 1.625$ \\
Disease knowledge & 8 & 80 & $57.56 \pm 19.426$ & $7.23 \pm 2.461$ \\
Coping ability after discharge & 3 & 30 & $22.85 \pm 6.268$ & $7.61 \pm 2.082$ \\
Available social support & 4 & 40 & $31.89 \pm 8.419$ & $8.04 \pm 2.111$ \\
Total score & 22 & 220 & $163.88 \pm 39.082$ & $7.39 \pm 1.763$ \\
\hline
\end{tabular}

Table 2. Discharge guidance quality scores of patients after cardiac valve surgery.

\begin{tabular}{ccccc}
\hline Dimension & No. of Entries & Full Score & Actual Score & Standardized Score \\
\hline Required content & 6 & 60 & $47.14 \pm 11.839$ & $7.86 \pm 1.973$ \\
Received content & 6 & 60 & $45.49 \pm 13.137$ & $6.50 \pm 1.876$ \\
Instructional skills and effects & 12 & 120 & $101.46 \pm 20.247$ & $8.46 \pm 1.687$ \\
Total score & 24 & 240 & $194.09 \pm 40.643$ & $8.09 \pm 1.694$ \\
\hline
\end{tabular}

Table 3. Comparison of discharge readiness scores of patients with different demographic characteristics after cardiac valve surgery.

\begin{tabular}{|c|c|c|c|c|}
\hline Item Entry & $\begin{array}{l}\text { Num. of } \\
\text { Cases (\%) }\end{array}$ & $\begin{array}{l}\text { Total Discharge } \\
\text { Readiness Score }\end{array}$ & $\mathrm{t} / \mathrm{F} / \mathrm{H}$ & $\mathrm{P}$ \\
\hline \multicolumn{5}{|l|}{ Gender } \\
\hline Male & 80 & $170.39 \pm 37.145$ & \multirow{2}{*}{2.446} & \multirow{2}{*}{$0.016^{*}$} \\
\hline Female & 50 & $153.48 \pm 40.214$ & & \\
\hline \multicolumn{5}{|l|}{ Age } \\
\hline$\leq 30$ years & 11 & $164.45 \pm 25.947$ & \multirow{3}{*}{0.002} & \multirow{3}{*}{0.998} \\
\hline $30-59$ years & 75 & $163.73 \pm 40.672$ & & \\
\hline$\geq 60$ years & 44 & $164.00 \pm 39.734$ & & \\
\hline \multicolumn{5}{|l|}{ Literacy level } \\
\hline Primary school & 30 & $167.73 \pm 44.909$ & \multirow{4}{*}{0.648} & \multirow{4}{*}{0.585} \\
\hline Junior high school & 43 & $167.35 \pm 37.530$ & & \\
\hline Technical secondary school/high school & 40 & $161.70 \pm 39.819$ & & \\
\hline College/undergraduate & 17 & $153.47 \pm 30.166$ & & \\
\hline \multicolumn{5}{|l|}{ Marital status } \\
\hline Married & 99 & $163.18 \pm 40.869$ & \multirow{3}{*}{0.267} & \multirow{3}{*}{0.766} \\
\hline Unmarried & 12 & $160.50 \pm 37.752$ & & \\
\hline Divorced/Widowed & 19 & $169.68 \pm 30.596$ & & \\
\hline \multicolumn{5}{|l|}{ Occupation } \\
\hline On-the-job & 53 & $159.68 \pm 45.245$ & \multirow{3}{*}{4.474} & \multirow{3}{*}{$0.013^{\star}$} \\
\hline Retired & 24 & $148.71 \pm 42.235$ & & \\
\hline Unemployed & 53 & $174.96 \pm 26.526$ & & \\
\hline \multicolumn{5}{|l|}{ Household monthly income per capita } \\
\hline$<2000$ yuan & 26 & $172.35 \pm 39.057$ & \multirow{5}{*}{1.164} & \multirow{5}{*}{0.330} \\
\hline $2000-3999$ & 40 & $162.48 \pm 37.793$ & & \\
\hline $4000-5999$ & 42 & $167.24 \pm 36.720$ & & \\
\hline $6000-9999$ & 17 & $147.59 \pm 49.276$ & & \\
\hline Above 10,000 & 5 & $158.40 \pm 22.289$ & & \\
\hline
\end{tabular}




\section{Continued}

Medical payment method

Own expense

Medical insurance

Cooperative Medical Care

Distance from home to hospital

Less than $1 \mathrm{~km}$

$1-4.9 \mathrm{~km}$

$5-10 \mathrm{~km}$

More than $10 \mathrm{~km}$

Habitation manner

Live alone

With spouse

With children

With spouse and children

Other

Type of primary caregiver

Spouse

Offspring

Spouse and offspring

Other relatives

Others hired

Destination after discharge

Back home

Transferred to a rehabilitation institution

Other

Way of Admission

Emergency

Elective

Other

Disease onset situation

Initial onset

Relapse

Number of hospitalizations due to the disease

1 time

2 times

3 times
13

65

52

$148.38 \pm 49.478$

$168.32 \pm 42.492$

$162.21 \pm 30.536$

$136.67 \pm 1.155$

$177.33 \pm 29.084$

$187.78 \pm 29.303$

$158.63 \pm 40.283$

$152.00 \pm 25.104$

$163.56 \pm 45.867$

$170.52 \pm 40.933$

$161.38 \pm 38.083$

$14 \quad 170.71 \pm 32.262$

$0.584 \quad 0.675$

$62 \quad 160.73 \pm 41.265$

$30 \quad 160.70 \pm 33.407$

$12 \quad 163.32 \pm 45.403$

$17 \quad 176.35 \pm 31.676$

$2 \quad 209.00 \pm 39.082$

1.261

0.289

$166.95 \pm 38.116$

$146.00 \pm 48.273$

$9 \quad 141.33 \pm 35.021$

2.756

0.067

$55 \quad 170.78 \pm 38.076$

$155.98 \pm 41.557$

$162.45 \pm 36.501$

1.759

0.176

$162.83 \pm 38.730$

$171.93 \pm 42.208$

$-0.847$

0.399

$64.46 \pm 39.562$

$37.409 \pm 6.613$

$206.00 \pm 8.718$

${ }^{*} \mathrm{p}<0.05$.

Table 4. Correlation analysis of discharge readiness and discharge guidance quality of patients after cardiac valve surgery.

\begin{tabular}{cccccc}
\hline & $\begin{array}{c}\text { Total score } \\
\text { for discharge } \\
\text { readiness }\end{array}$ & Self-condition & $\begin{array}{c}\text { Disease } \\
\text { knowledge }\end{array}$ & $\begin{array}{c}\text { Coping } \\
\text { ability after } \\
\text { discharge }\end{array}$ & $\begin{array}{c}\text { Available } \\
\text { social } \\
\text { support }\end{array}$ \\
\hline $\begin{array}{c}\text { Total Discharge Teaching } \\
\text { Quality Score }\end{array}$ & $0.602^{* *}$ & $0.414^{* *}$ & $0.608^{* *}$ & $0.541^{* *}$ & $0.438^{* *}$ \\
Required content & $0.384^{* *}$ & $0.244^{* *}$ & $0.383^{* *}$ & $0.376^{* *}$ & $0.294^{* *}$ \\
Received content & $0.487^{* *}$ & $0.266^{* *}$ & $0.583^{* *}$ & $0.374^{* *}$ & $0.283^{* *}$ \\
Instructional skills and effects & $0.668^{* *}$ & $0.515^{* *}$ & $0.618^{* *}$ & $0.623^{* *}$ & $0.525^{* *}$ \\
\hline
\end{tabular}




\subsection{Analysis of Influencing Factors of Patient Discharge Readiness after Cardiac Valve Surgery}

Taking discharge readiness as the dependent variable, the factors that presented statistical significance in the previous one-way analysis of variance and the quality of discharge guidance as the independent variables, multiple linear regression analyses were performed. The results are shown below in Table 5 .

\section{Discussion}

\subsection{The Patients' Readiness for Discharge after Valve Replacement Was at a Moderate Level}

The average score of each item of hospital discharge readiness after CVR was $7.39 \pm 1.763$ points, which was at a moderate level relative to the full 10 points. With 7 points being the criterion, only 50 patients $(38.46 \%)$ had an average score of $\geq 7$ points. Valve replacement, coronary artery bypass grafting, large vessel surgery, and congenital heart disease are common procedures in cardiac surgery. Compared with other cardiac surgery patients, the patients in this study were less prepared to discharge from the hospital than patients with coronary heart disease in the study of Wang et al. [11] (8.01 points), while basically the same as patients with with aortic dissection in the research of Guo et al. [12] (7.36 points). This result could be related to the following factors: the average age of the patients in this study was 53.08 years old, and there were 91 cases $(70 \%)$ of elderly patients over 50 years old, which led to reduced patient tolerance. In addition, most CVR procedures belong to open thoracotomy with cardiopulmonary bypass, which brings long operation time and massive surgical trauma, and thus requires long recovery period. Furthermore, more than $90 \%$ of patients undergoing valve replacement require life-long regular anticoagulation therapy. The management of anticoagulant drugs after discharge requires frequent rechecking of coagulation indicators and adjustment of anticoagulant doses. The cumbersome management of anticoagulation may be one of the factors affecting the readiness for discharge. Lastly, most valve replacement patients have experienced a chronic course of disease onset, and most of the patients' heart function is already in the decompensated stage even before the operation, which is different from the rapid recovery of the acute disease situation. The recovery of such patients' heart function requires a certain period of time after the operation.

Table 5. Multiple linear regression analysis of readiness for discharge of patients after cardiac valve surgery.

\begin{tabular}{ccccc}
\hline Variable order & & $\mathrm{R}$ & $\mathrm{R}^{2}$ & $\mathrm{p}$ \\
\hline Constant term & 70.310 & & & 0.000 \\
$X_{1}$ Guidance quality total score & 0.531 & 0.602 & 0.363 & 0.000 \\
$X_{2}$ Gender & -18.861 & 0.622 & 0.387 & 0.002 \\
$X_{3}$ Occupation & 8.329 & 0.645 & 0.417 & 0.012 \\
\hline
\end{tabular}


In summary, the aforementioned points are all factors that affect the readiness for discharge of patients undergoing valve replacement surgery.

After valve replacement, the scores of the various dimensions of the patient's readiness to discharge from the hospital, from high to low, were available social support, coping ability after discharge, self-condition, and disease knowledge, respectively. The patients' self-condition and disease knowledge scores were low, indicating that the patients were still in the recovery stage rather than fully recovered when discharged from the hospital, and had not yet mastered sufficient disease-related knowledge. This is inconsistent with the research results of other scholars. In Guo et al. [12] and other studies, the average discharge readiness scores of patients with aortic dissection were ranked from high to low, with seld-condition being the highest, followed by coping ability after discharge, disease knowledge, and available social support. The reasons for the differences in the analysis could be as follows: in this study, CVR patients had chronic disease course in the past, so a relatively stable family support system has been established in the course of the disease, and certain knowledge has also been accumulated during the hospitalization process. The relatively comprehensive discharge plan of our department also improved the patient's self-confidence in the adaptation ability after discharge, so the patient's coping ability after discharge was also in a relatively positive state. Nevertheless, the chronic disease course is also a long-term consumption process, so the feeling of the patients' own condition would be lower than that of the other research's arterial dissection patients who mainly suffered from acute disease. The disease knowledge dimension had the lowest score. In conversations with patients, it was found that self-management of anticoagulant drugs could be the biggest problem that confused the patients, indicating that the education and guidance of anticoagulation management for CVR patients are very important. From the beginning of the patient's operation, a variety of methods and channels should be used to guide and teach anticoagulation management. It is the key to the success of post-operative home anticoagulation management to pay attention to the feedback of patients and address patients' questions in a timely manner. It is worth noting that the item with the lowest score in the entire questionnaire was "health facilities and information that can be provided in your community after returning home", with only 5.96 points, which reveals the contradiction between patients' need of community medical information for the sake of continued care after discharge and the relatively scarce medical resources in the community.

\subsection{Quality of Discharge Guidance for Patients after CVR}

In this study, the quality of patient discharge guidance was at the upper-moderate level $(8.09 \pm 1.694)$, and the difference between the scores of patient's required content $(7.86 \pm 1.973)$ and received content $(6.50 \pm 1.876)$ was negative, indicating that most patients received discharge guidance that did not meet their expectations. The dimensional scores of instructional skills and effects were at a 
high level $(8.46 \pm 1.687)$, and the analyzed reasons are as follows: patients had high expectations of the nurses, while the nurses' levels of nursing profession and discharge guidance still need to be improved; the cultural awareness and medical knowledge in the nurse-patient interaction culture were highly asymmetrical, and there was a difference in the assessment of readiness for discharge between the two; the patients' sense of participation in preparations for discharge was not strong, while the formality and content of the nurses' guidance still need to be improved. In view of the current promotion and application of the concept of Fast-Track Surgery and the increase in hospital bed turnover rate, the average hospital stay of patients undergoing CVR surgery has been significantly shortened. Under such a medical situation, it is worthwhile to explore how to ensure the safety of the discharged patients while assuring the capability of the patients and their families regarding follow-up care. It is indicated that nursing work should also consider the variety of health education formalities [13], such as multidisciplinary teamwork, mind mapping, multimedia information technology intervention, and unobstructed informational channels after discharge. The goal is to improve the quality of patient health education and increase the patient's readiness and confidence in hospital discharge.

\subsection{The Correlation between the Hospital Discharge Readiness of Patients after Valve Replacement and the Quality of Discharge Guidance}

The results of this study showed that there was a positive correlation between the discharge readiness score and the discharge guidance quality score of patients after valve replacement, which is consistent with the results of other scholars. Therefore, attention should be paid to the discharge guidance of CVR patients to ensure that the patients are in a better state of preparation for discharge. The training of specialist nurses should be strengthened to improve the effect of patient discharge guidance. At the same time, patients with different cultural backgrounds should be encouraged to participate in the joint decision-making of the discharge preparation plan.

\subsection{Influencing Factors of Patient Discharge Readiness after Valve Replacement}

The results of international studies on the influencing factors of hospital discharge readiness [14] [15] [16] [17] [18] showed that the influencing factors of patients' discharge readiness mainly involve the patient's personal characteristics, therapeutic care measures, disease factors and social support. In this study, gender, occupation, and the distance between home and hospital were the influencing factors for the readiness of CVR patients to discharge. In a study of patients with fragility fractures, Lu et al. [19] found that gender was a factor affecting the readiness for discharge. Consistent with the results of this study, the readiness for discharge of male patients was better than that of female patients. It might be related to the traditional concept that female in the family take on 
more roles as caregivers and need to consider more issues than male patients. Occupational status was also a factor influencing the readiness for discharge of CVR patients. Retired people had the lowest readiness; those who had work ranked in the middle, while those who were unemployed had the highest readiness. This is inconsistent with the results of Wang et al. [11] on patients with coronary heart disease. Wang et al.'s research showed that unemployed people had the lowest readiness for discharge from hospital, and retired people had the highest readiness. The reason for the discrepancy may be related to the educational structure. In Wang et al.'s study, 94.5\% of the unemployed patients had a junior high school literacy level or below. In this study, only $45 \%$ of the unemployed patients had junior high school or lower education level. This resulted in insufficient ability to accept and understand information, leading to a lower perception of readiness for discharge. In this study, the average age of the retired population was $67 \pm 20.17$ years old, and $92 \%$ of the population had an educational background below junior high school. The elder age and low educational structure led to a lower perception of readiness for discharge. This study also found that the distance between home and hospital was also an influencing factor of CVR patients' discharge readiness. In general, the closer the home was to the hospital, the better the patient's readiness for discharge. This could be because the closer the home is to the hospital, the more convenient it is for patients to obtain medical resources, and the patients feel safer psychologically.

\section{Conclusion}

This survey study showed that CVR patients' discharge readiness and the quality of discharge guidance were at moderate levels, and the quality of discharge guidance positively affects the discharge readiness of patients. Therefore, in clinical work, attention should be paid to patient discharge guidance, personalized health education should be implemented, and an evaluation system suitable for discharge readiness of CVR patients should be explored to improve the quality of patient guidance. A comprehensive and effective assessment of the readiness for discharge of CVR patients is the basis for the implementation of discharge management, which can ensure that patients are discharged in the best condition and at the most appropriate time, and reduce the incidence of complications and readmission rates after discharge. It can not only save medical resources and reduce medical expenses, but it is also particularly important for CVR patients' self-management after discharge.

\section{Limitations}

This study only investigated post-CVR patients in one hospital. Considering one hospital and one department's specific management system, policies and other specific influences, the study presented certain limitations. It is suggested that multi-center research can be carried out in the future to make the results more representative. 


\section{Fund}

This research was funded by the Nursing Research Fund of the Third Affiliated Hospital of Sun Yat-sen University (2019HL04).

\section{Conflicts of Interest}

The authors declare no conflicts of interest regarding the publication of this paper.

\section{References}

[1] Zhang, B.R. and Xu, Z.Y. (2007) Valvular Heart Surgery. People's Medical Publishing House, Beijing.

[2] Bezuidenhout, D., Williams, D.F. and Zilla, P. (2015) Polymeric Heart Valves for Surgical Implantation, Catheter-Based Technologies and Heart Assist Devices. Biomaterials, 36, 6-25. https://doi.org/10.1016/j.biomaterials.2014.09.013

[3] Yan, H.Q. and He, J.C. (2014) Current Situation and Development of Treatment of Valvular Heart Disease. Fujian Journal of Medicine, 3, 140-141.

[4] Li, B., Duo, L.L., Yu, Y.Y., et al. (2014) The Effect of Transitional Care on the Anticoagulant Therapy Compliance among Discharged Patients Undergoing Cardiac Valve Replacement Surgery. Chinese Nursing Management, 14, 1278-1281.

[5] Lua, D., Padwal, R.S., Majumdar, S.R., et al. (2016) Patient-Reported Discharge Readiness and 30-Day Risk of Readmission or Death: A Prospective Cohort Study. The American Journal of Medicine, 129, 89-95. https://doi.org/10.1016/j.amjmed.2015.08.018

[6] Fenwick, A.M. (1979) An Interdisciplinary Tool for Assessing Patients' Readiness for Discharge in the Rehabilitation Setting. Journal of Advanced Nursing, 4, 9-21. https://doi.org/10.1111/j.1365-2648.1979.tb02984.x

[7] Galvin, E.C., Wills, T. and Coffey, A. (2017) Readiness for Hospital Discharge: A Concept Analysis. Journal of Advanced Nursing, 73, 2547-2557. https://doi.org/10.1111/jan.13324

[8] Marianne, E., Weiss, M.E. and Linda, B. (2006) Psychometric Properties of the Readiness for Hospital Discharge Scale. Journal of Nursing Measurement, 14, 163-178. https://doi.org/10.1891/jnm-v14i3a002

[9] Zhao, H., Feng, X., Yu, R., Gu, D., and Ji, X. (2015) Validation of the Chinese Version of the Readiness for Hospital Discharge Scale on Patients Who Have Undergone Laryngectomy. The Journal of Nursing Research, 24, 321-328. https://doi.org/10.1097/JNR.0000000000000126

[10] Wang, B.H., Wang, H. and Yang, C.Z. (2016) Reliability and Validity of the Chinese Version of the Quality of Discharge Teaching Scale. Chinese Journal of Nursing, 51, 752-755.

[11] Wang, B.H., Wang, H., Yang, C.Z., et al. (2017) Survey of the Readiness for Hospital Discharge and Its Influencing Factors among Coronary Heart Disease Patients. Chinese Journal of Nursing, 52, 138-143.

[12] Guo, W.P., Zhang, Q. and Su, L. (2018) Influencing Factors of Discharge Readiness for Patients after Aortic Dissection Surgery. PLA Nursing Journal, 35, 18-22.

[13] Liang, L.M., Pan, Y.H., Wu, D.C., et al. (2019) Effects of Multidisciplinary Team-Based Nurse-Led Transitional Care on Clinical Outcomes and Quality of Life in Patients with Ankylosing Spondylitis. Asian Nursing Research, 13, 107-114. 
https://doi.org/10.1016/j.anr.2019.02.004

[14] Knier, S., Stichler, J.F., Ferber, L., et al. (2015) Patients' Perceptions of the Quality of Discharge Teaching and Readiness for Discharge. Rehabilitation Nursing Journal, 40, 30-39. https://doi.org/10.1002/rnj.164

[15] La Manna, J.B., Bushy, A., Norris, A.E., et al. (2016) Early and Intermediate Hospital-to-Home Transition Outcomes of Older Adults Diagnosed with Diabetes. The Diabetes Educator, 42, 72-86. https://doi.org/10.1177/0145721715620020

[16] Healy, S.J., Black, D., Harris, C., et al. (2013) Inpatient Diabetes Education Is Associated Withe Less Frequent Hospital Readmission among Patients with Poor Glycemic Control. Diabetes Care, 36, 2960-2967. https://doi.org/10.2337/dc13-0108

[17] Maniar, H.S., Bell, J.M., Moon, M.R., et al. (2014) Prospective Evaluation of Patients Readmitted after Cardiac Surgery: Analysis of Outcomes and Desertification of Outcomes and Identification of Risk Factors. The Journal of Thoracic and Cardiovascular Surgery, 147, 1013-1020. https://doi.org/10.1016/j.jtcvs.2013.10.066

[18] Rochefort, C.M., Rathwell, B.A. and Clarke, S.P. (2016) Rationing of Nursing Care Interventions and Its Association with Nurse-Reported Outcomes in the Neonatal Intensive Care Unit: Across-Sectional Survey. BMC Nursing, 15, 46-53.

https://doi.org/10.1186/s12912-016-0169-Z

[19] Lu, F.J., Song, Y.Z. and Liu, C.C. (2019) Survey of the Readiness for Hospital Discharge and Its Influencing Factors among Osteoporotic Fracture Patients. Journal of Nurse Development, 34, 294-313. 